Bundesgesundheitsbl - Gesundheitsforsch Gesundheitsschutz 2006 • 49:833-835 DOI 10.1007/s00103-006-0014-8 C) Springer Medizin Verlag 2006

\title{
Parameter des roten Blutbildes bei Exposition durch Mobilfunkanlagen
}

\section{Mitteilung der Kommission „Methoden und Qualitätssicherung in der Umweltmedizin“}

- Es fehlen Messwerte der Feldstärken und Angaben zu Zeitspannen zwischen Exposition und Blutentnahme, so dass eine Quantifizierung der „Mobilfunkbelastung“ fehlt.

- Die statistischen Berechnungen sind an vielen Stellen unvollständig bzw. fehlerhaft.

Weitere Anmerkungen zu methodischen Aspekten dieser Arbeit sind veröffentlicht worden [2]. "Geldrollenbildung" ist ein weiteres, teilweise mit Live-Vorführungen in der Öffentlichkeit demonstriertes Phänomen („Vitalblut-Diagnostik“), das im Zusammenhang mit Mobilfunk aufgegriffen und als brisant angesehen wird [3].

Die RKI-Kommission „Methoden und Qualitätssicherung in der Umweltmedizin“ nimmt im Folgenden zum diagnostischen Wert der Bestimmung der Anzahl der Retikulozyten im peripheren Blut und zum Phänomen der Geldrollenbildung im Zusammenhang mit der Exposition gegenüber elektromagnetischen Feldern Stellung.

\section{Retikulozyten}

Retikulozyten entstehen im Knochenmark aus orthochromen Erythroblasten und sind die Vorstufe der Erythrozyten. Sie enthalten während ihrer etwa viertägigen Reifungsphase im Unterschied zu den reifen Erythrozyten noch RNA und Organellen. Ihre Ribosomen sind netzartig miteinander verbunden und geben den Retikulozyten ihren Namen. Der Anteil der Retikulozyten liegt bei Erwachsenen zwischen 0,5 und 2\% der Erythrozyten, bei Neugeborenen deutlich höher bei o,6-6\% (Normwerte). Für Männer und Frauen werden unterschiedliche Referenzbereiche angegeben. Bei pathologischen Störungen der Erythropoese, nach Erythropoietingabe (Doping) und in Folge einer Exposition gegenüber ionisierender Strahlung kann die Anzahl erhöht oder erniedrigt sein. Die Messung der Anzahl der Retikulozyten ist allein nicht ausreichend, um pathologische Veränderungen der Hämatopoese zu diagnostizieren. Zumindest müssen gleichzeitig der Hämoglobingehalt der Retikulozyten, die Anzahl der Erythrozyten und der Hämatokrit bestimmt werden [4].

\section{Die Bestimmung der Retikulozytenzahl}

Retikulozyten werden durch RNA-spezifische Farbstoffe (z. B. durch BrillantKresylblau) angefärbt und anschließend mikroskopisch gezählt oder heute in der Regel automatisch mittels Durchflusszytometer und Laser-Streulicht bestimmt. Der Variationskoeffizient der mikroskopischen Auszählung beträgt 17-54\% [5]. Die automatisierten Verfahren zeichnen sich gegenüber der manuellen Methode durch eine deutlich geringere Streuung aus. Dies ist u. a. durch die Objektivierung der Messung und die 
größere Anzahl analysierter Zellen (oft 10.000 Zellen gegenüber 100o) bedingt. Aufgrund der Intensität der Fluoreszenz, die dem RNA-Gehalt proportional ist, können die Retikulozyten klassifiziert und weitere Indizes dieses Zelltyps bestimmt werden (z.B. Volumen $(\mathrm{MCVr})$, Hämoglobin-Gehalt $(\mathrm{CHr})$ und -Konzentration (CHCMr)) [6]. Es ist viel Erfahrung notwendig, um Retikulozyten richtig zu bestimmen und die Befunde zu bewerten.

Die Anzahl der Retikulozyten kann physiologisch schwanken, ohne dass dies ein Hinweis auf eine Erkrankung sein muss. Selbst Blutproben ein und derselben Person sind hinsichtlich der Anzahl der Retikulozyten in bedeutendem Umfang variabel $[7,8]$. Der InterPersonen-Variationskoeffizient liegt bei automatisierten Verfahren bei 26 bis 33\%, der Intra-Personen-Koeffizient bei etwa 11\%. Die Zellindizes der Retikulozyten hingegen schwanken wesentlich weniger mit Inter-Personen-Variationen von unter $5 \%[8]$.

\section{Geldrollenbildung}

Als „Geldrollenbildung“, auch RouleauBildung oder Pseudoagglutination (engl. red blood cell aggregation, blood sludge oder pearl chains, rouleaux formation) genannt, wird die perlschnurartige Aneinanderreihung von Erythrozyten bezeichnet. Sie wird durch Adhäsionskräfte zwischen Erythrozyten verursacht, ist an der Regulierung der Druckverhältnisse in Venolen beteiligt [9] und kann bei Doppleruntersuchungen ein Hintergrundrauschen verursachen $[10,11]$. Sie ist normalerweise reversibel. Verschiedene Faktoren können die Rollenbildung beeinflussen wie Alter, Dehydrierung, Fließgeschwindigkeit, Hypoxie, Blutfettgehalte, Gerinnungsstatus und Arzneimittel $[12,13,14,15,16]$ und modulieren damit die Fließeigenschaften des Blutes.

Die Tendenz zur Geldrollenbildung wird an frisch entnommenem Blut untersucht [17]. Die klassische Methodik ist die mikroskopische Auswertung. Sie hat den Vorteil, dass die Ergebnisse direkt sichtbar sind, und den Nachteil, dass sie im Vergleich zu neuen Methoden wie der Laser-Refraktometrie [18] weniger objektiv ist. Da die Geldrollenbildung an frischem Blut durch Temperatur, Inkubationsmedium, mechanische Einflüsse und andere Faktoren beeinflusst wird, muss in jedem Fall ein hoher Grad an qualitätssichernden Maßnahmen und Standardisierung eingehalten werden. Gerade Untersuchungen, bei denen geringfügige Veränderungen noch erfasst werden sollen, bedürfen stringenter Standardisierung, um Artefakte und Fehlinterpretationen zu vermeiden [19]. Dies ist bei Felduntersuchungen oft nicht gegeben [20].

\section{Bewertung und Empfehlung}

Anders als für Effekte ionisierender Strahlung [7], deren Wirkungen auf das blutbildende System von erheblicher pathologischer und diagnostischer Bedeutung sind, ist die Bestimmung der Retikulozytenzahl zum Nachweis von Effekten infolge Einwirkung EMF nicht geeignet. Es liegen bislang keine wissenschaftlichen Studien vor, die einen Einfluss schwacher EMF der Mobilfunktechnologie auf Retikulozyten und Erythrozyten nachweisen und die anhand valider und reliabler Messverfahren das Phänomen der Geldrollenbildung auf EMF-Einfluss zurückführen könnten. Dies gilt sowohl für Basisstationen, deren Feldstärken im Aufenthaltsbereich der Bevölkerung so gering sind, dass sie keine thermischen Effekte verursachen, als auch für Endgeräte, deren Gebrauch lokal begrenzte Erwärmungen nach sich ziehen können. Davon abgesehen ist die alleinige Bestimmung der Anzahl der Retikulozyten aufgrund der hohen Streuung als Messgröße ungeeignet. Insgesamt ist die Bestimmung von Retikulozyten und/ oder Geldrollenbildung als biologischer Marker für eine Mobilfunkbelastung allein schon wegen der hohen intraund interindividuellen Variabilität nicht geeignet.

\section{Einstufung}

Die Bestimmung der Retikulozyten im peripheren Blut oder von Geldrollenbildung wird sowohl im Hinblick auf die umweltmedizinische Einzelfall-
Diagnostik als auch im Kontext von Gruppenuntersuchungen in die Kategorie IV eingestuft [21]: Die Bestimmungen können für den klinisch-umweltmedizinischen Bereich nicht empfohlen werden, da die Messergebnisse keine sinnvollen Aussagen oder Rückschlüsse auf biologische Wirkungen von EMF erlauben. Die seitens der Befürworter solcher Untersuchungen postulierten Gründe für die Indikation von Reihenuntersuchungen bei MobilfunkExponierten sind spekulativ und basieren nicht auf einem validierten diagnostischen Ansatz.

\section{Federführung}

Prof. Dr. A. Lerchl (externer Sachverständiger, International University Bremen (IUB), School of Engineering and Science, Research II, Campus Ring 6, 28759 Bremen)

Dr. med. B. Heinzow (Mitglied der Kommission, Landesamt für Gesundheit und Arbeitssicherheit des Landes Schleswig-Holstein, Dezernat 50, Umweltbezogener Gesundheitsschutz, Brunswickerstr. 4, 24105 Kiel)

\section{Weitere von der Kommission hinzugezogene externe Sachverständige}

Dr. S. Heller (St. Gertrauden Krankenhaus, Zentrallabor, Paretzer Str. 11-2, 10713 Berlin)

Prof. Dr. med. R. Averdunk, Herzzentrum Berlin, Labor, 13353 Berlin)

\section{RKI-Kommission „Methoden und Qualitätssicherung in der Umweltmedizin"}

\section{Mitglieder}

\section{Kommissionsmitglieder}

Prof. Dr. rer. nat. W. Dott (Universitätsklinikum Aachen, Institut für Hygiene und Umweltmedizin), Prof. Dr. med. H. Drexler (Friedrich-AlexanderUniversität Erlangen-Nürnberg, Institut für Arbeits-, Sozial- und Umweltmedizin), Prof. Dr. med. H. Dunkelberg (Universität Göttingen, Abt. Allg. Hygiene und Umweltmedizin), Prof. Dr. med. Th. Eikmann (Universität Gießen, Institut für Hygiene und Umweltmedizin), Dr. med. 
B. Heinzow (Landesamt für Gesundheit und Arbeitssicherheit des Landes Schleswig-Holstein, Dezernat Umweltbezogener Gesundheitsschutz, Kiel), Prof. Dr. C. Hornberg (Universität Bielefeld, Fakultät für Gesundheitswissenschaften), Institut für Innenraum- und Umwelttoxikologie), Prof. Dr. med. K. E. von Mühlendahl (Kinderhospital Osnabrück, Gemeinnützige Kinderumwelt $\mathrm{GmbH}$ ), Prof. Dr. med. D. Nowak (LMU München, Klinikum Innenstadt, Institut und Poliklinik für Arbeits- und Umweltmedizin), PD Dr. med. F.-A. Pitten (Institut für Krankenhaushygiene und Infektionskontrolle GbR, Gießen), Dr. med. W. Stück (Ökologischer Ärztebund/ISDE, Koblenz), Prof. Dr. M. Schwenk (Tübingen), Dr. med. R. Suchenwirth (Niedersächsisches Landesgesundheitsamt, Abt. Umweltmedizin/Epidemiologie, Hannover; zz. Ministerium für Soziales, Frauen, Familie und Gesundheit, Hannover), Prof. Dr. med. M. Wilhelm (Ruhr-Universität Bochum, Hygiene, Sozial- und Umweltmedizin).

\section{Ständige Gäste}

S. Strecker (Bundesministerium für Gesundheit, Bonn), Dr. med. N. Englert (Umweltbundesamt, Berlin)

\section{Geschäftsstelle im RKI}

Dr. med. D. Eis (Geschäftsführer), Dr. med. U. Wolf.

\section{Literatur}

1. German P (2004) „Einfluss der Mobilfunkbelastung auf die Retikulozytenreifung - Vorläufige Bewertung anhand von 1000 Analysen". Die Studie war ursprünglich abrufbar unter http://www. die-umwelt-akademie.de/veranstaltungen/T54c. html, ist aber inzwischen vom Server genommen worden. Eine weitere Quelle ist http://www. buergerwelle.de/pdf/einfluss_mobilfunk_auf_ retikulocyten_juli04.doc

2. Lerchl A (2005) Blutuntersuchungen in der EMF-Forschung: Dichtung und Wahrheit. FGF newsletter 2/2004. www.fgf.de/fup/ themen/inhalte-themenforum/ NL_03-04/ Blutuntersuchungen_in_der_EMF_Forschung_ 03-04d.pdf

3. Ritter M, Wolski W (2005) Geldrollenbildung durch Handystrahlung, Jugend Forscht. http:// www.milieuziektes.nl/Rapporten/20050325_ Geldrollenbildung_durch_Handystrahlung.pdf

4. Brugnara C (1998) Use of reticulocyte cellular indices in the diagnosis and treatment of hematological disorders. Int J Clin Lab Res 28: 1-11
5. Deiss A, Kurth D (1970) Circulating reticulocytes in normal adults as determined by the new methylene blue method. Am J Clin Pathol 53: 481484

6. Siekmeier R, Bierlich A, Jaross W (2000) Determination of reticulocytes: three methods compared. Clin Chem Lab Med 38: 245-249

7. Möning H, Pohlit W, Sattler EL (1993) Biologische Dosimetrie. Zivilschutzforschung, Schriftenreihe der Schutzkommission beim Bundesminister des Inneren Bd. 12, Hrsg. Bundesamt für Zivilschutz

8. Sandberg S, Rustad P, Johannesen B, Stolsnes $B$ (1998) Within-subject biological variation of reticulocytes and reticulocyte-derived parameters. Eur J Haematol 61: 42-48

9. Kim S, Popel AS, Intaglietta M, Johnson PC (2005) Aggregate formation of erythrocytes in postcapillary venules. Am J Physiol Heart Circ Physiol 288: H584-590

10. Black IW (2000) Spontaneous echo contrast: where there's smoke there's fire. Echocardiography 17: 373-382

11. Welsh AW, Rubin J, Fowlkes J, Fisk N (2005) Standardization of power Doppler quantification of blood flow in the human fetus using the aorta and inferior vena cava. Ultrasound Obstetrics Gynecology 26: 33-43

12. Cokelet GR, Goldsmith HL (1991) Decreased hydrodynamic resistence in the two-phase flow of blood through small vertical tubes at low flow rates. Circ Res 68: 1-17

13. Cicha I, Suzuki Y, Tateishi N, Maeda N (2003) Changes of RBC aggregation in oxygenationdeoxygenation. $\mathrm{pH}$ dependency and cell morphology. Am J Physiol Heart Circ Physiol 284: 2335-2342

14. Cicha I, Suzuki Y, Tateishi N, Maeda N (2004) Effects of dietary triglycerides in rheological properties of human red blood cells. Clin Hemorheol Microcirc 30: 301-305

15. Ben-Ami R, Barshtein G, Zeltser D et al. (2001) Parameters of red blood cell aggregation as correlates of the inflammatory state. Am J Physiol Heart Circ Physiol 280: 1982-1988

16. Muravyov AV, Yakusevich VV, Kabanov AV, Petrochenko AS (2005) The effect of diuretics on red blood cell microrheological parameters in female hypertensive patients. Clin Hemorheol Microcirc 33: 121-126

17. Rampling MW, Whittingstall P (1986) A comparison of five methods for estimating red cell aggregation. Klin Wochenschr 64: 1084-1088

18. Hardeman MR, Dobbe JG, Ince C (2001) The laser-assisted rotational cell analyzer (LORCA) as red blood cell aggregometer. Clin Hemorheol Microcirc 25: 1-11

19. Löffler H, Rastetter J, Haferlach T (2004) Atlas der klinischen Hämatologie, 6. Aufl. Springer, Berlin Heidelberg New York Tokyo

20. Gollnick F, Conrad G (2000) Geldrollenbildung im Blut verursacht durch Mobilfunkfelder. FGF newsletter 1:4-7. http://www.fgf.de/fup/publikat/ news_einzel/NL_00-01/GeldrollenbildungMobilfunk-NL01_00d.pdf

21. RKI (2001) Grundsätze der Bewertung von umweltmedizinischen Methoden. Mitteilung der Kommission „Methoden und Qualitätssicherung in der Umweltmedizin" Bundesgesundheitsbl Gesundheitsforsch Gesundheitsschutz 44: 519-522 